\title{
LIMITES DE LA MÉTHODE DE RECONNAISSANCE DE L'ORIGINE (MARINE OU DULCICOLE) DE LA TRUITE (SALMO TRUTTA L.) AU MOYEN DE L'ANALYSE DE LA TENEUR EN STRONTIUM DES ECAILLES
}

\author{
par G. MAISSE et J. L. BAGLINIERE \\ INRA, Station de Physiologie et d'Ecologie des Poissons \\ 35042 RENNES Cedex
}

\section{RÉSUMÉ}

La teneur en strontium des écailles de truites, d'origines anadrome et dulcicole, capturées dans des rivières de Basse-Normandie, dépend du réseau hydrographique, du temps de séjour en mer et de la date de capture. L'influence de ce dernier facteur est probablement liée à la maturation sexuelle.

\section{LIMITS OF METHOD OF DISCRIMINATION BETWEEN SEAWATER AND FRESHWATER ORIGINS OF TROUT (SALMO TRUTTA L.) USING THE SCALE STRONTIUM LEVEL}

\section{SUMMARY}

Strontium content of scales of anadromous and freshwater trout caught in rivers in Basse-Normandie, varies with drainage basin, time spent in the sea and date of capture. The influence of this last factor is probably in relation to sexual maturation.

\section{INTRODUCTION}

L'extrême plasticité de l'espèce Truite (Salmo trutta L.) entraîne son adaptation à des milieux différents (rivière, lac, estuaire, mer) ; ainsi dans la plupart des fleuves côtiers se jetant dans la Manche et dans l'Atlantique coexistent des truites de mer et des truites sédentaires qui peuvent être difficiles à distinguer (ARRIGNON, 1967). Généralement, une analyse scalimétrique classique (RICHARD et BAGLINIĖRE, 1990) portant sur l'étude de la disposition des circuli, permet de retracer la vie des individus et en particulier leur passage en mer où leur croissance est la plus importante. Cependant, dans les grands fleuves, les truites peuvent trouver des conditions trophiques très favorables comparables à celles des grands lacs, voire de la mer. Dans ce cas les écailles des diverses origines présentent tellement de similitudes qu'il est difficile de trancher. BAGENAL et al., 1973, confrontés au même problème ont testé une méthode basée sur l'analyse de la teneur en strontium des écailles ; la discrimination entre des truites capturées en mer et d'autres prises en eau douce a été possible, les premières ayant des niveaux supérieurs à $300 \mu \mathrm{g} / \mathrm{g}$ et les secondes des niveaux inférieurs à $200 \mu \mathrm{g} / \mathrm{g}$. De même, PINAULT, SALIOU, GERARD et MAISSE (données non publiées) ont noté des niveaux moyens de $600 \mu \mathrm{g} / \mathrm{g}(\mathrm{n}=20, \sigma=66)$ pour des truites élevées en mer depuis 18 mois et de $215 \mu \mathrm{g} / \mathrm{g}(n=9, \sigma=13)$ pour des truites élevées en eau douce. Le problème qui nous est posé est cependant quelque peu différent par le fait que l'ensemble des truites à analyser sont capturées en rivière, ce qui pourrait avoir une répercussion sur la teneur en strontium des écailles des individus d'origine marine.

D'une manière globale, les teneurs en strontium des écailles et de l'os operculaire sont très voisines (MOREAU et al., 1983), mais il est probable que, compte tenu de sa taille, ce dernier permettrait une analyse plus fine. Cependant, le choix des écailles plutôt qu'une autre structure osseuse a été dicté par deux impératifs: la nécessité de garder vivantes la majorité des truites étudiées et le refus des pêcheurs à la ligne de procéder à une mutilation nuisant à l'aspect de leur capture. 


\section{MATÉRIEL ET MÉTHODE}

Les truites étudiées provenaient de deux rivières de Basse-Normandie, la Calonne, affluent de la Touques, et l'Oir, affluent de la Sélune. Capturées dans des pièges lors de leur migration avant ou après le frai, elles ont été classées en trois types, "truites de rivière", "finnock" (individu remontant en rivière l'année de sa première dévalaison en mer), "truite de mer" (individu ayant passé au moins un hiver en mer), d'après leur livrée et l'interprétation scalimètrique proposée par RICHARD et BAGLINIĖRE (1990).

Le dosage du strontium a été réalisé sur $5 \mathrm{mg}$ d'écailles suivant la méthode décrite par BAGENAL et al., (1973) en utilisant un spectrophotomètre à absorption atomique, l'étalonnage externe étant établi avec des solutions standard.

\section{RÉSULTATS (Tableau I)}

Les résultats enregistrés sur les truites de l'Oir montrent qu'à l'automne, il est impossible de reconnaître l'origine des individus capturés pendant la migration vers les zones de frai, à partir de la teneur en strontium de leurs écailles. Au printemps, après le frai, les truites de mer et les finnocks ont des niveaux semblables à ceux des poissons de remontée.

Sur la Calonne, les finnocks ont des niveaux comparables en été et en automne, alors que pour les truites de mer, le niveau estival est significativement (test $T, p<0,001$ ) plus élevé que le niveau automnal. Par ailleurs, en été, la teneur moyenne est significativement (test $T, p<0,001$ ) plus élevée chez les truites de mer que chez les finnocks.

A l'automne, les niveaux relevés chez les finnocks et les truites de mer sont significativement (test $T$, respectivement $p<0,01$ et $p<0,001$ ) plus élevés sur l'Oir que sur la Calonne.

Tableau I : Moyennes des teneurs en strontium des écailles de truite suivant la
rivière, la saison de capture et le type de poisson.

Table I : Means of Sr-content in scales of trout according to the river, the catching season and type of fish.

\begin{tabular}{|c|c|c|c|}
\hline Rivière & Saison & $\begin{array}{l}\text { Type de } \\
\text { poisson }\end{array}$ & $\operatorname{Sr}(\mu \mathrm{g} / \mathrm{g})$ \\
\hline \multirow{5}{*}{ Oir } & \multirow{3}{*}{$\begin{array}{c}\text { Automne } \\
\text { (montaison) }\end{array}$} & $\begin{array}{c}\text { Truite } \\
\text { de rivière }\end{array}$ & $\begin{array}{c}247 \\
n=18, \sigma=53\end{array}$ \\
\hline & & Finnock & $\begin{array}{c}238 \\
n=13, \sigma=84\end{array}$ \\
\hline & & $\begin{array}{l}\text { Truite } \\
\text { de mer }\end{array}$ & $\begin{array}{c}220 \\
n=15, \sigma=34\end{array}$ \\
\hline & \multirow{2}{*}{$\begin{array}{l}\text { Printemps } \\
\text { (avalaison) }\end{array}$} & Finnock & $\mathrm{n}=21, \sigma=56$ \\
\hline & & $\begin{array}{l}\text { Truite } \\
\text { de mer }\end{array}$ & $\begin{array}{c}239 \\
n=12, \sigma=66\end{array}$ \\
\hline \multirow{4}{*}{ Calonne } & \multirow{2}{*}{$\begin{array}{c}\text { Été } \\
\text { (montaison) }\end{array}$} & Finnock & $\begin{array}{c}179 \\
n=20, \sigma=38\end{array}$ \\
\hline & & $\begin{array}{l}\text { Truite } \\
\text { de mer }\end{array}$ & $\begin{array}{c}265 \\
\mathrm{n}=19, \sigma=74\end{array}$ \\
\hline & \multirow{2}{*}{$\begin{array}{c}\text { Automne } \\
\text { (montaison) }\end{array}$} & Finnock & $\begin{array}{c}176 \\
\mathrm{n}=20, \sigma=72\end{array}$ \\
\hline & & $\begin{array}{l}\text { Truite } \\
\text { de mer }\end{array}$ & $\begin{array}{c}155 \\
\mathrm{n}=20, \sigma=54\end{array}$ \\
\hline
\end{tabular}




\section{DISCUSSION ET CONCLUSION}

Nos observations montrent que la méthode de reconnaissance de l'origine, marine ou dulcicole, des truites, à partir de la teneur des écailles en strontium, doit être utilisée avec précaution lorsqu'on s'intéresse à des individus capturés en rivière. II apparaît que le niveau de strontium dépend au moins de trois facteurs : le réseau hydrographique, le temps de séjour en mer, la saison de capture.

Le premier de ces facteurs avait déjà été mis en évidence par MOREAU et al. (1983) chez l'omble de fontaine (Salvelinus fontinalis) et par GAUSEN et BERG (1988) chez des smolts de saumon atlantique (Salmo salar L.).

Le facteur "temps de séjour en mer" qui apparaît chez les individus capturés en été, recouvre probablement outre le facteur temps, la salinité et l'alimentation. L'influence de ce dernier point est bien connu à partir des expérimentations de marquage chimique réalisées en pisciculture avec des aliments enrichis en strontium (OPHEL et JUDD, 1968, chez Carassius auratus, BEHRENS YAMADA et al., 1979, chez Oncorhynchus Kisutch, GUILLOU et de LA NOUE, 1987, chez Salvelinus fontinalis). Si le temps de séjour en mer est le premier point qui différencie les finnocks des truites de mer, il est possible que les premiers se cantonnent aux estuaires avec une salinité et une alimentation différentes de celles rencontrées par les truites de mer.

Le facteur "saison de capture" est important pour les truites de mer. Ce facteur peut être associé au temps passé par les poissons depuis leur retour en rivière jusqu'au moment de leur capture. Cependant, CASTONGUAY et FITZGERALD (1982) ont montré que des ombles de fontaine élevés en eau salée (20 pour mille) conservaient une teneur en strontium caractéristique d'un séjour en mer, pendant au moins 2,5 mois. Dans le cas des truites de mer de Basse-Normandie nous pensons que la gamétogénèse est probablement un des facteurs responsables de la chute du niveau de strontium enregistré dans les écailles entre l'été et l'automne ; la mobilisation des réserves minérales à ce moment du cycle sexuel est bien connue chez les salmonidés et se traduit au niveau de la périphérie des écailles par une érosion physiologique qui, selon son intensité, donne naissance à une marque de frai plus ou moins prononcée. Cette mobilisation préférentielle des réserves minérales du pourtour de l'écaille va détruire en premier lieu la zone la plus jeune et en particulier pour les truites de mer, celle formée pendant la vie marine, contenant la plus forte teneur en strontium (BAGENAL et al., 1973).

En conclusion, il apparaît à la lumière de cette étude que la distinction de l'origine anadrome ou dulcicole de truites capturées en rivière n'est pas possible en automne et au printemps sur les individus ayant frayé, à partir de la seule analyse du strontium contenu dans les écailles. En été, seules les truites ayant passé au moins un hiver en mer (type "truite de mer") se démarquent des autres types. Un effet "rivière" très important existe et implique la nécessité de procéder à une analyse par bassin.

\section{REMERCIEMENTS}

Cette étude a été financée en partie par le Conseil Supérieur de la Pêche. Les analyses du strontium ont été réalisées au Laboratoire associé INRA de Méthodologie Diagnostique de l'Ecole Nationale Vétérinaire de Nantes par Madame Martine LEBLOIS, sous la responsabilité du Professeur $L$. PINAULT. Les prélèvements d'écailles ont été réalisés par G. LECLERC à la station de Pont-l'Evêque (Fédération des AAPP du Calvados), F. MARCHAND et R. DELANOÉ à la station de Cerisel (Fédération des AAPP de la Manche).

\section{BIBLIOGRAPHIE}

ARRIGNON J., 1967. Comportement de l'espèce "Salmo trutta" dans le bassin de la Seine. Bull. Fr. Piscic., 227, 56-71.

BAGENAL T.B., MACKERETH F.J.H., HERON J., 1973. The distinction between brown trout and sea trout by the strontium content of their scales. J. Fish Biol., 5, 555-557.

BEHRENS YAMADA S., MULLIGAN T.J., FAIRCHILD S.J., 1979. Strontium marking of hatchery-reared coho salmon (Oncorhynchus kisutch, Walbaum). J. Fish Biol., 14, 267-275. 
CASTONGUAY M., FITZGERALD G.J., 1982. Critique de la méthode de distinction entre poissons anadromes et dulcicoles de la même espèce par la teneur en strontium de leurs écailles. Can. J. Fish. Aquat. Sci., 39, 1423-1425.

GAUSEN D., BERG O.K., 1988. Strontium levels in scales and vertebrae of wild Atlantic salmon, Salmo salar L., smolts. Aqua. Fish. Managt., 19, 299-304.

GUILLOU A., DE LA NOUE J., 1987. Use of strontium as a nutritional marker for farmreared brook trout. Prog. Fish-Cult., 49, 34-39.

MOREAU G., BARBEAU C., FRENETTE J.J., SAINT-ONGE J., SIMONEAU M., 1983. Zinc, Manganese and strontium in opercula and scales of brook trout (Salvelinus fontinalis) as indicators of lake acidification. Can. J. Fish. Aquat. Sci., 40, 1685-1691.

OPHEL I.L., JUDD J.M., 1968. Marking fish with stable strontium. J. Fish. Res. Bd. Canada, 25, 1333-1337.

RICHARD A., BAGLINIĖRE J.L., 1990. Description et interprétation des écailles de truites de mer (Salmo trutta L.) de deux rivières de Basse-Normandie : I'Orne et la Touques. Bull. Fr. Pêche Piscic., Spec. coll. (sous presse). 